\title{
Restrictions on therapists
}

\section{Despite the approval of the General Dental Council to therapists being allowed to work in all sectors of dentistry, they have been excluded from the changes in expanding the dental team}

Mike Grace m.grace.bdj@bda-dentistry.org.uk

\begin{abstract}
The apparently inexplicable decision by the Privy Council to prevent therapists from expanding their current duties to enable them to work in general dental practice has created an atmosphere of indignation and outrage among both the therapists themselves and a substantial number of dentists.
\end{abstract}

The story began in March when the Privy Council rejected an amendment to the Dental Auxiliaries Regulations which would have allowed therapists to work in all sectors of dentistry. The reason given for this decision was that the amendment would have provided a disproportionate benefit to dentists employing therapists under the General Dental Services (GDS) present remuneration system. This seems a somewhat unusual excuse in light of the current arrangement where hygienists can work in the GDS, apparently without providing a similar 'disproportionate benefit'.

Dental therapists provide an obvious and valuable benefit in the treatment of patients. However, despite their obvious abilities for contributing to general dental care, they have always suffered from some major restrictions that appear somewhat 'draconian'. The first of these is that therapists can only carry out conservative treatment within the National Health Service. This is stated in the Dentist's Act 1984 as "no auxiliary can carry out "the filling of teeth or the extraction of deciduous teeth" except in the course of provision of national health services'. It is interesting to speculate why therapists are the only dental health workers with this legal restriction.

The second restriction is that therapists were originally only allowed to work in the community services. This was relaxed slightly in 1998 with the introduction of the Personal Dental Services (PDS) where therapists were allowed to work in general practice within a PDS scheme, but only because the PDS is considered to be an extension of the CDS. This means that a dentist working in general practice within a PDS scheme can employ a therapist, but he/she cannot treat patients privately nor can he/she treat GDS patients. One wonders at the morality (as well as the legality) of this situation. The final restriction on therapists is this latest decision. Despite the approval of the General Dental Council to therapists being allowed to work in all sectors of dentistry, they have been excluded from the changes in expanding the dental team. This decision was apparently taken after the Privy Council had taken the advice of the Department of Health. Where is the sense in all of this?

The current GDS is both underfunded and stretched, and we all know that the Prime Minister has promised to deliver NHS dentistry to all by September 2001. Only 300 of the 1,300 qualified therapists are currently working because there are not enough jobs for them in the existing community services and PDS pilot schemes. There is a shortage of family dentists and some patients are experiencing difficulty in obtaining access to NHS dentistry. Many therapists already work in general practice because they have retrained as hygienists because of the difficulty in obtaining work. The answer seems obvious. The Privy Council should rethink their decision and listen to other views. The Department of Health's apparent concern that therapists would be 'drained off' from the community service seems unlikely in view of the facts stated above, and both dentists and therapists are united in their wish to be able to increase the provision of NHS dentistry for their patients. If this were not to happen then it would suggest a deeper hidden agenda to the use of therapists - or perhaps to the intention to replace the GDS with the PDS in the not-too-distant future. If that were the plan (always denied officially) then a strategy of restricting therapists to the CDS and PDS only would make perfect sense. 